\title{
Quality Improvement in Hospitals: Identifying and Understanding Behaviors
}

\author{
Lukasz M. Mazur ${ }^{1}$, John K. McCreery ${ }^{2}$, Shi-Jie (Gary) Chen ${ }^{3}$ \\ ${ }^{1}$ Department of Radiation Oncology, School of Medicine, University of North \\ Carolina, NC, USA \\ ${ }^{2}$ Poole College of Management, North Carolina State University, Raleigh, NC, USA \\ ${ }^{3}$ Industrial and Systems Engineering, Northern Illinois University, DeKalb, IL, USA
}

Submitted November 2011. Accepted for publication September 2012.

\begin{abstract}
Improving operational performance in hospitals is complicated, particularly if process improvement requires complex behavioral changes. Using single-loop and double-loop learning theory as a foundation, the purpose of this research is to empirically uncover key improvement behaviors and the factors that may be associated with such behaviors in hospitals. A two-phased approach was taken to collect data regarding improvement behaviors and associated factors, and data analysis was conducted using methods proposed by grounded theorists. The contributions of this research are twofold. First, five key behaviors related to process improvement are identified, namely Quick Fixing, Initiating, Conforming, Expediting, and Enhancing. Second, based on these observed behaviors, a set of force field diagrams is developed to structure and organize possible factors that are important to consider when attempting to change improvement behaviors. This begins to fill the gap in the knowledge about what factors drive effective improvement efforts in hospital settings.
\end{abstract}

Keywords: quality improvement, patient safety, behavior change

\section{INTRODUCTION}

Since the Institute of Medicine's landmark 1999 report, To Err Is Human [1], revealed the widespread incidence of medical errors in U.S. hospitals, there has been a great deal of effort to improve the quality and patient safety of healthcare delivery systems. Much progress has been made in developing policies, quality models, indicators, best practices, and risk-adjustment mechanisms to report and compare improvement efforts across institutions, and in examining practices and cultures in high-performing hospitals [2-6]. While these types of improvement efforts have proliferated during the last

*Corresponding Author: Lukasz M. Mazur, Ph.D., Department of Radiation Oncology, School of Medicine, University of North Carolina, Chapel Hill, NC 27599. Phone: (919) 616-9702. Email: 1mazur@med.unc.edu. Other authors: mccreery@ncsu.edu; garychen@niu.edu. 
decade, progress toward reliable and safe healthcare delivery systems in hospitals remains unacceptable [7-9]. Hospitals in general are still plagued by cultural and attitudinal challenges that act as barriers to making needed improvements [10].

For decades, thought leaders in healthcare improvement have suggested that U.S. hospitals would benefit from implementing improvement initiatives supported by process reengineering principles, with leadership committed to a culture of safety and teamwork [11-19]. Studies of high reliability organizations and organizations with mature quality and safety programs have confirmed such approaches and further emphasized the importance of developing and empowering frontline employees to detect and eliminate process failures [20-26].

Improvement efforts in hospitals are affected by a broad range of contextual factors while undergoing process changes [27-35]. Recent studies of barriers and facilitators from the quality improvement perspective highlight a variety of factors at the individual and organizational levels. Solomons and Spross [36] used Shortell's [37] framework of continuous quality improvement to identify barriers and facilitators to Evidence Based Practice. Their comprehensive literature search identified the following factors that may contribute to suboptimal behavioral change: time constraints, lack of support, lack of reward systems, staffing issues, heavy workloads, resistance to change, lack of authority and autonomy, and insufficient training. Other studies found organization change, leadership style and behavior, organizational culture, data infrastructure, information systems, and years involved in quality improvement as the key contextual factors that contribute to success or failure in quality improvement initiatives [38]. Researchers also identified physician involvement, microsystem motivation to change, resources for quality improvement, and quality improvement team leadership as additional contributing factors [39].

Reid and Catchpole [40], while recognizing that factors like reliable systems and well designed policies and work processes are important, also noted that the greatest factors contributing to unsafe behavior appear to be "frailties of the human condition, complacent attitudes and unconscious behaviors" of frontline caregivers. Accordingly, there is a need for more empirical research to gain a deeper understanding of caregiver improvement behaviors. Without an understanding of the content and context of these behaviors, it will be difficult to achieve breakthrough results over the long haul. The purpose of our research is to identify key improvement behaviors for healthcare frontline professionals and to gain insights into the key factors that drive these behaviors. We hope that our findings may help equip improvement practitioners and leaders with knowledge on how to pragmatically guide their employees toward necessary behavioral changes in order to improve quality, reduce cost, and increase patient safety.

\section{THEORETICAL FOUNDATION}

Our choice of theoretical foundation is grounded in a belief that healthcare improvement is a form of experiential learning in which effectiveness depends on the ability to change human behavior [41-42]. As such, we recognize that factors associated with improvement behaviors are grounded in how individuals self-regulate their actions [43]. In this context, we study quality improvement behaviors using the 
single-loop and double-loop learning models proposed by Argyris and Schon [44]. These models were utilized by the National Health Services in England to explore individual and organizational learning and the characteristics of the organizational cultures needed to underpin such learning [45-46].

According to Argyris and Schon [44], individuals engaging in single-loop learning place importance on the efficiency and effectiveness of addressing the negative consequences of their actions, but not on fundamental values that govern their behaviors that propelled their actions in the first place. Individuals that utilize singleloop learning often perform learning in isolation without access to all relevant information and limited self-reflection regarding their behaviors. While single-loop learning tends to temporarily adjust one's behavior, double-loop learning tries to identify, resolve, and positively change the fundamental values that govern such behavior. Compared with single-loop learning, double-loop learning is more thoughtful and reflective. Thus, a question of obvious importance is: What are the critical factors that might guide or drive improvement behaviors based on second-loop learning in organizations?

First, research has shown that individuals who engage in improvement behaviors using double-loop learning must feel competent and be motivated to utilize various problem-solving techniques to remove the root causes of problems [47]. Second, individuals must also feel psychologically safe to share their opinions in an open and honest manner, must have access to valid information, and must be provided with sufficient time to apply their knowledge to initiate problem solving efforts [48]. Third, the visible, active involvement of direct managers in root-cause problem solving efforts has been shown to clarify improvement behavior roles, increase feelings of competence, and help create a culture that values continuous improvement efforts [49]. Fourth, research also suggests that organizational support and constructive feedback can stimulate individuals' beliefs that organizational processes and resources needed to tackle identified problems exist and are actually used to solve problems. Furthermore, there is evidence that feedback can enhance an individual's sense of psychological safety if it demonstrates that engaging in improvement behaviors is safe for interpersonal risk-taking [50]. Finally, literature indicates that individuals must feel a certain level of job-related autonomy, as it decreases burnout [51-52] and promotes self-management, which accordingly increases individual motivation to make decisions regarding productivity [53-54].

While the concepts of single-loop and double-loop learning are somewhat familiar in the management literature, they have received surprisingly little attention in the context of improvement behaviors in healthcare delivery [55]. Moreover, healthcare literature offers limited empirical findings regarding Argyris and Schon's theory, thus motivating our exploratory and investigative efforts for this study.

\section{METHODS}

This research effort is structured as two distinct but related phases. As shown in Figure 1, we performed a set of activities linked to a single community hospital, followed by a subsequent set of activities carried out with four rural hospitals. 


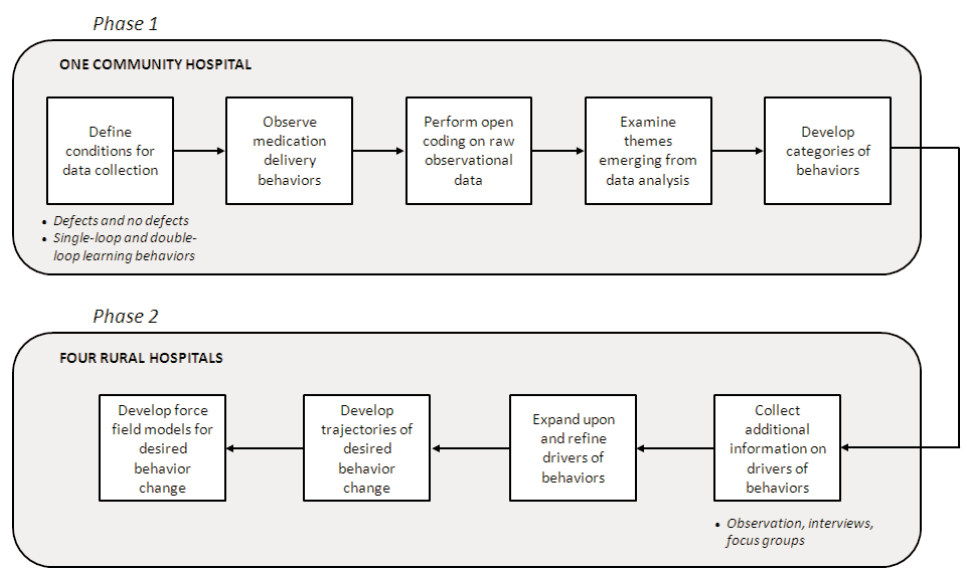

Figure 1. Research methodology.

The initial phase of our study took us to a community hospital to observe behaviors. We wanted a setting within this hospital that was relatively complex and challenging, and therefore exhibited inherent variability in execution, but had standard procedures in place for how to perform the work. This would allow us to collect a rich set of exploratory data on behaviors, but stay consistent in terms of the context of the observations. After examining options that would meet these criteria, we chose to observe the medication delivery process.

We collected data over an interval of two years by periodically observing individuals in 12 different departments in the hospital [56]. The departments involved were the Catheterization Lab, Cancer Treatment Center, Labor and Delivery, Nursery, Post Anesthesia Care Unit, Day Surgery, Emergency Department, Operating Room, Intensive Care Unit, Medical Floor, Surgical Floor, and Pharmacy (with five subsystems, namely, medication delivery, IV delivery, narcotics delivery 1 , narcotics delivery 2, and purchasing). The subjects were 63 nurses and 6 pharmacy technicians with an average experience of approximately eight years. Our sample represented $7 \%$ of the entire full time employee population at the community hospital.

Participation in the research study was voluntary with all nurses and technicians offered participation via email which included a description of the study and the consent form. All participants were familiar with and had at least a basic level of competency with the Plan-Do-Check-Act cycle, which was the hospital's basic continuous quality improvement methodology.

All observation-related data were recorded on standard data collection sheets by a member of the research team during continuous observation periods ranging from two to eight hours. All study subjects consented to be 'shadowed' with a stated goal to observe medication delivery processes. The subjects were not necessarily cognizant of the detailed research objectives regarding improvement behaviors and factors that drive these behaviors. Qualitative data were collected from participants who, on a voluntary 
basis, provided verbal feedback on their actions as well as related issues that they considered relevant. This verbal feedback was subsequently crosschecked for accuracy and sound interpretation with the study subjects.

Since our objective is to understand behaviors related to quality improvement, we considered the question of when an improvement opportunity can occur. Our observations and data collection efforts were designed to cover situations when defects were present as well as situations without defects, as opportunities for improvement exist whether a defect is present or not [21,37]. For our purposes, a defect is defined as a condition where the individual is attempting to perform a task and needs something that is unavailable or defective, and/or something is present that should not be present $[25,49]$, resulting in a failure to execute the task as intended. We define a defect-free condition as one where an individual is attempting to perform a task and everything needed is available, and nothing significant is present that should not be present [23, 47]. When defect-free, the task can be performed as designed and intended.

We first initiated a process of coding using a grounded theory approach proposed by Strauss and Corbin [57] to search for high level themes associated with participants' verbal responses. Because most study participants usually engaged in more than one type of improvement behavior, it is important to note that our intent was to examine and understand improvement behaviors and search for common themes related to these behaviors. It was not our intent to classify people and their verbal responses into specific improvement categories. Members of the research team, while meeting face-toface, analyzed the observational and interview data to ensure proper interpretation.

A number of themes emerged from an initial review of the data. The participants gave voice to a wide range of concerns as they encountered improvement opportunities. In addition, we were able to identify distinct categories of behavior that were useful in understanding how individuals may act when opportunities for improvement exist. While valuable insights were gained from the community hospital study, and will be discussed in the Results section of this paper, we concluded that more investigation of this research problem was warranted to obtain a deeper understanding of the key factors that drive these behaviors.

Therefore, we developed a second phase to this research, collecting additional supporting information from a wide range of employees at four rural hospitals to augment and refine the research findings. In this phase, we did not code for improvement behaviors. Instead, we conducted a total of 96 hours of direct observations of improvement events, 16 hours of semi-structured 1-hour interviews with 16 different hospital leaders, and an additional 3 hours of focus groups with 8-12 individuals per group, including directors, managers, and frontline professionals. Standard questions used as prompts during interviews with leaders and focus groups are presented in Appendix A. Two members of the research team conducted each of the leader interviews, with one person asking questions and the second person capturing responses using hand-written notes. Focus groups were spearheaded by one researcher, who asked and noted responses concurrently. These hospitals were members of a collaborative network to implement the Toyota Production System or 'Lean' methodology for continuous quality improvement and to share good implementation 
practices. This phase of the research provided critical insights and allowed us to refine our findings from phase 1 in four primary ways. First, it allowed us to bring forward the themes and behaviors from phase 1, refining them through further observation, discussion, and reflection. Second, the interactions in phase 2 provided new insights and new information related to improvement behaviors and possible drivers of these behaviors. Third, it allowed us to link the categories of improvement behaviors in a meaningful way, to make them more applicable and relevant to healthcare organizations that are focused on developing double-loop learning capabilities. And fourth, we were able to develop a scheme for organizing and explaining managerial challenges related to the wide range of possible drivers of improvement behavior. We employed Force Field Analysis [58-59] as an organizing technique, which has been used in a variety of contexts and has been shown to assist leaders in implementing positive change in their organizations.

The research methods of this study preserved the participants' anonymity and confidentiality of responses and were approved by the local Institutional Review Board (IRB) to ensure appropriateness for both academia and the participating hospitals.

\section{RESULTS}

This section is organized into three parts. In section 4.1, we present preliminary themes that emerged from analysis of verbal feedback collected in phase 1, while in Section 4.2 we present and explain five distinct categories of improvement behavior that cover the range of our observations and are consistent with single- and double-loop learning theory. In section 4.3, we identify and organize a set of factors that may possibly move frontline professionals from less desirable behaviors to more desirable behaviors.

\subsection{Themes of Observed Behaviors}

A wide range of improvement behavior was observed at the community hospital. From an analysis of the verbal responses, we found four emergent themes. They are described in the following sections 4.1.1 to 4.1.4.

\subsubsection{Training for Improvement}

Most study participants at one point in time tried to engage in some sort of improvement actions. Individuals often voiced their personal frustrations and disengagement from improvement behaviors as a result of poor training. Specifically, 52 nurses ( $83 \%$ of the total) talked about poor training on how to report defects, and 48 nurses $(76 \%)$ indicated difficulties with utilizing improvement tools and methods. All pharmacy technicians pointed to these same issues. For example:

"I always try to improve or do things better, like other techs in our department. But I think I had ten minutes of training on how to use our system for reporting and improving medication errors. That is all."

"Our system for reporting and improvement of medication errors is not userfriendly at all! It asks for so much detail. First of all I don't have time to do it, but second of all, it is just too frustrating to fill out all this information." 


\subsubsection{Time for Improvement}

All nurses and all technicians highlighted productivity pressures and low staffing levels as the most stress-generating factors in their daily work. This led to many comments concerning the use of workarounds and shortcuts.

"Every day I do a lot of workarounds. Everyone does it. We need to get things done. I could do it according to the procedure, but, I don't know, this is just quicker and saves me some time."

We took note of nurses and technicians often seeing themselves as not having the time to resolve underlying causes of problems that arise in daily activities. Nurses in particular often voiced concerns about spending a significant proportion of their time performing ancillary services. Consequently, we were not surprised that both nurses and pharmacy technicians were hardly able to keep up with their job duties and were in essence forced to quickly patch problems to manage their workloads.

\subsubsection{Feedback from Improvement}

Fifty four nurses (86\%) and 4 out of 6 technicians specified lack of feedback as another major contributing factor that decreases the reporting, analysis, and improvement rates of errors. Also, nurses and technicians indicated that they are rarely involved in meetings regarding the improvement strategies proposed to offset defects. For example:

"I report medication errors. However, there is no feedback. It's like reporting to a black box! It is so frustrating, I can't even tell you."

"Some feedback about error reporting would be nice. I often wonder what is going on with all this data about medication errors."

The comments and discussions on feedback went beyond the specifics of reporting on medication errors. There was general concern that all layers of management, from direct supervisors to senior leadership, were not providing meaningful feedback on improvement actions taken by frontline staff. This can be summed up by the following comment.

"I don't expect any rewards for reporting or improving. All I want is feedback."

\subsubsection{Roles and Pressures for Improvement}

A number of distinct outcomes emerged that relate to roles and expectations of frontline professionals. We found that 55 nurses $(87 \%)$ and all of the technicians reported some level of confusion about their roles with respect to improvement efforts. It was not clear how they were to perform, what was expected of them, and at times who was accountable for the various activities that occur in a quality improvement environment. Given a strong organizational culture that values patient safety and excellent patient care, frontline caregivers at times found themselves trapped between conflicting expectations. While it was clear to all that the patient comes first, nurses and technicians 
also felt pressure to conform to existing, standard work procedures. When work procedures were perceived as being at odds with a patient-first approach, there was little guidance given to caregivers on how to proceed.

"For me, patient needs always come first. You must understand that I care for the patient's physiological, emotional, and even spiritual needs. We don't produce widgets here. When needed, I must make decisions to either follow procedures, which are often broken, or provide timely care for my patients. Do you see my dilemma?"

This type of pressure was exacerbated when a caregiver's actions were perceived as having ripple effects that could affect co-workers and others in the organization as well as the patient.

In addition, 44 nurses (70\%) and 3 out of 6 technicians suggested group and peer pressure as major factors affecting the willingness to comply with defect reporting expectations and participate in improvement efforts. Thirty one nurses self-reported poor compliance with defect reporting procedures due to group behavior influences. This is consistent with the Institute of Medicine report [6] which showed that failures in procedural compliance account for approximately three-quarters of all medication delivery errors.

"In our department we are all doing it differently [referring to error reporting and
making improvements]. Everyone has a different view about what I should do.....
What's expected of me? I try to do my job as well as I can. From time to time I
even suggest improvements to my manager or other nurses. But who else should
I talk to about my ideas?"

\subsection{Categories of Behaviors}

Of a total of 504 instances of improvement behavior, in 106 instances subjects operated under the condition of a system compromised by defects. Under such conditions, 94 times subjects behaved as single-loop learners. Only 12 times the subjects engaged in activity that could be described as double-loop learning. In addition, we observed 398 instances of subjects operating in a system free of defects, with subjects engaged 374 and 24 times in single-loop and double-loop learning behaviors, respectively.

For defect behavior, we found that when the system is compromised by defects, individuals will try to: (1) quickly fix the problems without addressing the underlying root causes, or (2) try to identify and initiate efforts to eliminate the root causes of problems. Alternately, when the system is not compromised by defects, individuals engage in one of the following three behaviors: (1) continue to conform to standard procedures and processes, (2) deviate from standard procedures and processes by taking shortcuts to get work done, without explicitly degrading operating performance or patient safety, or (3) in the spirit of continuous improvement, seek to make permanent enhancements to work processes and activities. These categories align with the ideas of single-loop and double-loop learning proposed by Argyris and Schon [42], which lends support to the appropriateness of our theoretical approach. Figure 2 and the subsequent bullets depict the categories of improvement behavior. 


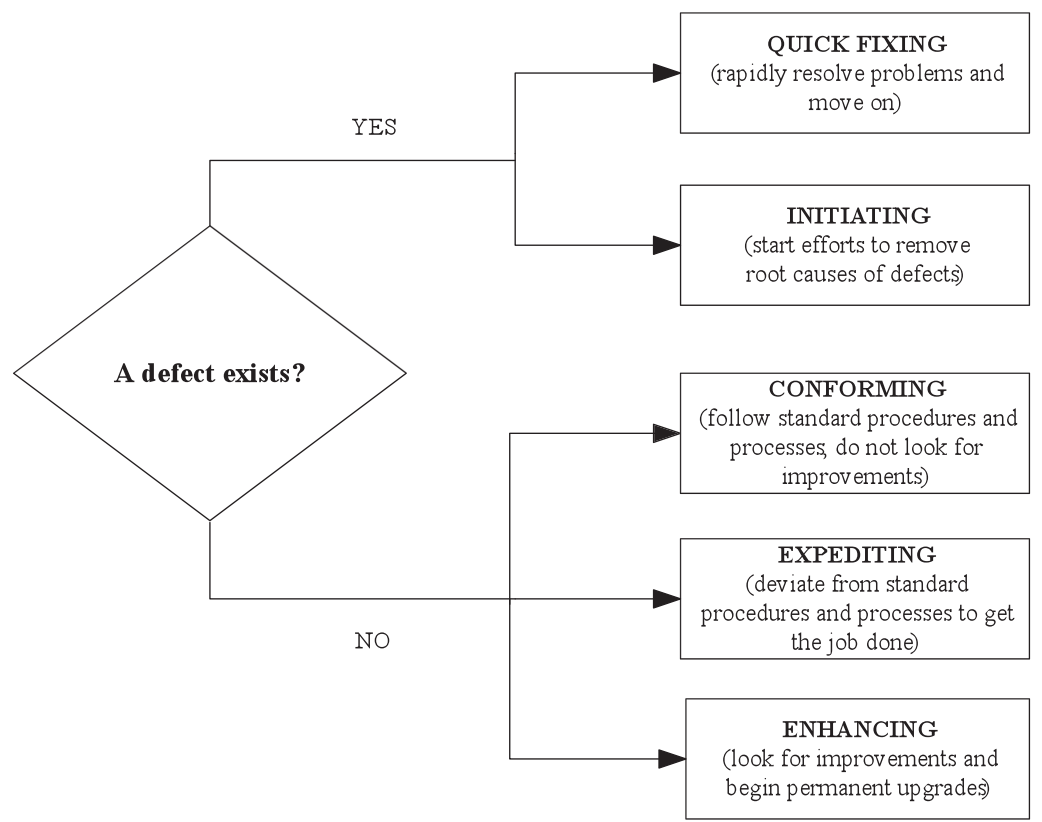

Figure 2. Categories of improvement behaviors.

- $\quad$ Quick Fixing - This behavior consists of detection and correction of defects often accompanied by a discussion or description - most often in the form of a complaint - of the problem to coworkers and the immediate manager, but without formal reporting of defects. In this behavior, importance is placed on the efficiency and effectiveness of localized fixing techniques, instead of analysis to remove the root cause(s) of the defects. Though the defect is recognized, the focus is on fixing what is wrong and moving on. For example, we observed a nurse faced with an absence of supplies needed for care delivery. She continued to search for the supplies throughout the department to fix the immediate crisis. The nurse never reported the problem or initiated an official request to remove the root cause(s) of the defects, but simply solved her immediate problem. This was a fairly common type of behavior, observed 94 times out of 504 observations in our study.

- Initiating - This behavior involves formal reporting of defects and the initiation of an improvement effort to improve the system. It also involves formal acknowledgment and action by the organization to correct the documented defects. In contrast to Quick Fixing, these behaviors are not motivated by short-term or immediate efficiency concerns but rather by longerterm goals to improve the system as a whole for the sake of patients or coworkers. We observed a nurse that engaged in Initiating while faced with the shortage of supplies needed to deliver care. She not only fixed the immediate problem of a lack of supplies, but also reported this defect and initiated improvement work to ensure that this problem would not recur. This type of behavior was observed less frequently, with subjects engaging only 12 times in Initiating behavior. 
- Conforming - This behavior is characterized by compliance with standard procedures and processes under the conditions of a system free of defects. Healthcare professionals engaged in Conforming behavior may be aware of the existing limitations or lack of perfection in the system, yet they are not willing to initiate improvement efforts in order to increase patient safety or system efficiency. To illustrate this type of behavior, we observed a nurse who worked on the unit that had only one dispensing machine on each wing, serving twelve patient rooms. At medication delivery time, which overlaps with shift change, physician rounds, and family visits, this nurse was instructed to dispense and administer additional medications to patients. This increase in workload caused some frustration in attempting to administer medications to patients on time. Afterward, the nurse did not initiate or engage in a system improvement effort to institute the appropriate countermeasures to remove the underlying root cause of this particular error. This was the most prevalent type of observed behavior, observed 272 times in our study.

- $\quad$ Expediting - This type of behavior describes non-compliant procedures performed to complete the work under the conditions of a system free of defects. Expediting behavior involves rule bending with the perceived (or misperceived) goal of providing timely or better care to patients. Thus, this behavior can be considered by employees as improvement behavior. For instance, we observed this behavior while a nurse tried to dispense medication for two patients at the same time, which was against the hospital's policy, in order to comply with timely medication administration requirements. Overall, subjects engaged in Expediting behaviors 102 times in our study.

- Enhancing - Enhancing behavior is seen in efforts to make long-lasting system improvements with regard to work efficiency, effectiveness, or patient safety, although the system is not overtly defective. The result of such behavior is the development of more efficient or safer work procedures to conduct care delivery tasks. We witnessed an instance where a nurse received a request to deliver additional medication to patients which took her beyond her normal workload. She understood that this request could lead to a defect, and so she used her personal and professional autonomy to stop the request. Further, she insisted on launching an improvement effort to uncover and remove the underlying root cause of this particular condition, where nurses are asked to deliver a higher volume of medications than acceptable. We witnessed such Enhancing behaviors in direct response to verbal orders for medication delivery given by physicians to nurses, under circumstances where written orders were required. Subjects were observed 24 times in Enhancing behavior.

The consequences to the organization of the five different behaviors are quite different. When defects occur, Quick Fixing behavior is effective at resolving immediate crises, but such efforts are not captured, validated, and disseminated by the organization to prevent recurrences in this or other units of the hospital. Thus, the challenge is to transform Quick Fixing behavior into Initiating behavior by calling attention to defects and providing the organization with the opportunity to take preventive action.

In the absence of defects, healthcare professionals can Conform, Expedite, or Enhance. The most desirable behavior is Enhancing, as this type of individual focuses 
on growing the organization's capabilities for efficient, high quality healthcare delivery. Such behaviors should be modeled and strongly supported by executive leadership and management. In contrast, Expediting behaviors involve shortcuts and deviations from standard operating procedures in order to make individuals' jobs easier or more productive, but the shortcuts cause problems of their own. Because of this noncompliance, Expediting behaviors may introduce variability and uncertainty into the system, which can cascade and cause downstream quality and safety problems. Thus, Expediting behaviors should be eliminated. The third type of non-defect behavior we observed is Conforming behavior. This type of individual is seemingly not motivated to make improvements to current ways of operating, since there are no overt defects to be dealt with. For the individual engaged in Conforming behavior, the logic of "leaving well enough alone" applies.

\subsection{Desired Behavior Change and Managerial Challenges}

As noted in the Methods section, the phase 2 effort with four rural hospitals allowed us to refine and expand upon phase 1 results. Figure 3 offers an integrated view of how both phases of this study come together to result in a usable set of behavior drivers.

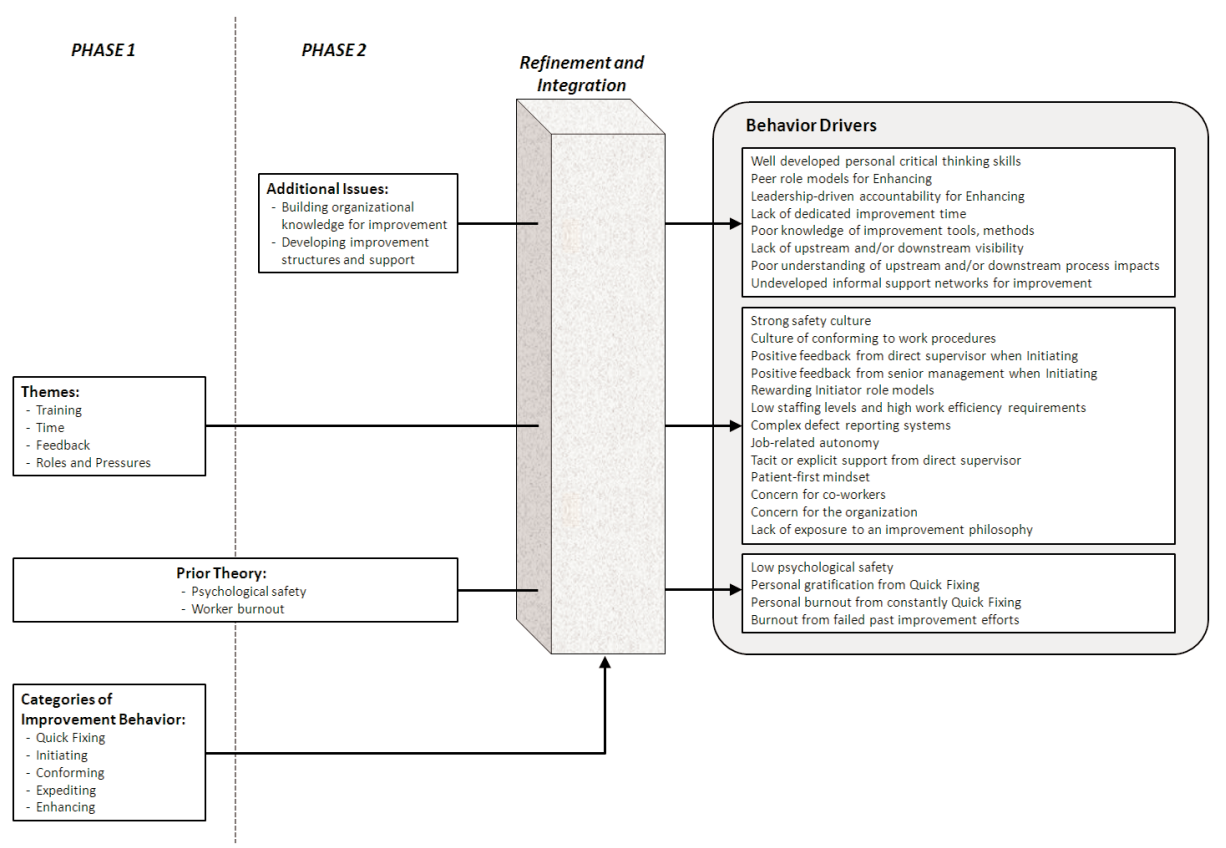

Figure 3. Sources of behavior drivers. 
As Figure 3 shows, both phases of the study provide inputs that ultimately result in a combined set of behavior drivers. In phase 1, a variety of important improvement behavior information was collected and initially grouped into the four high level themes of training, time, feedback, and roles and pressures. In addition, phase 1 allowed us to define five distinct categories of improvement behavior. In phase 2, the extensive observations, interviews, and focus groups uncovered two additional high level issues or themes: building organizational knowledge for improvement, and developing improvement structures and support. We believe these issues emerged in phase 2 for two reasons. First, they are primarily organizational-level issues, as opposed to being at the level of the individual. While subjects in phase 1 were individual frontline caregivers, phase 2 broadened the range of participants to include supervisors, managers, and senior leaders. The two organizational-level issues of phase 2 are rightly the concern of those higher up in the organization's hierarchy. Second, the rural hospitals in phase 2 were all implementing formalized organization-wide lean improvement programs, and programs such as these need organization-level structures and support.

Figure 3 also shows that prior theory on the topics of psychological safety and worker burnout were employed when developing the set of behavior drivers. Hence, data and insights from phase 1 , phase 2 , and prior literature were integrated and refined, using the five behavior categories to guide the development of behavior drivers shown on the right side of Figure 3.

Once the set of behavior drivers was identified, the next necessary step was to organize them in a meaningful way. Given the desirability of certain types of improvement behaviors - Initiating and Enhancing - over the other three types, we relied significantly on interactions with hospital executive leaders to help refine our thinking and to present the evidence in the format of managerial challenges. Through these interactions, two primary challenges emerged. One is the challenge of moving healthcare frontline professionals from Quick Fixing behavior to Initiating behavior when defects occur. The other key challenge is to move these same professionals toward continuous improvement Enhancing behavior, whether they are currently more complacent and using Conforming behaviors or using shortcut-taking Expediting behaviors. We organized our findings in the next sections to address these challenges and offer actionable recommendations for making progress in quality improvement.

\subsubsection{Challenge \#1: Transitioning from Quick Fixing to Initiating}

This challenge is one of how individuals will respond to defects when they occur. It is important to note that this is not a question of whether they will respond to defects when they are detected. Our observations strongly support the position that, by and large, healthcare professionals will not let defects occur without doing something. They are dedicated employees who take pride in their jobs and care about their patients. However, Quick Fixing and Initiating behaviors are both possible responses to a defect. An objective of our research is to provide insights on how to encourage and lead more employees in organizations to be initiators rather than quick fixers.

Developing Initiating behavior is the responsibility of both the individual and the organization. When defects occur, employees must be willing and able to initiate long 
term, root-cause improvements to processes and work practices. They must see Initiating behavior as a desirable response to take in the face of defects, even though it may require their investment of time and effort to accomplish. Likewise, the hospital organization must create an environment that fosters and rewards this type of behavior.

Figure 4 presents the driving forces and restraining forces for changing employee behavior from Quick Fixing to Initiating. Driving forces are presented on the left side of the figure, and represent the variety of significant factors that have emerged as drivers of the change from Quick Fixing to Initiating behavior. The right side of the figure presents the restraining forces that act to keep healthcare professionals acting in Quick Fixing mode.

The good news is that in our research, we have noticed a number of factors that can drive the change to Initiating behavior. The top two on the left side of the figure - a strong safety culture and a culture of conforming to work procedures - are organization level factors that, in tandem, can drive employees to exhibit Initiating behavior. If there is a strong culture of safety in a hospital, then employees are motivated to operate first and foremost with the objective of patient care. Excessive bureaucracy and unnecessary rules and procedures will be subordinated to actions that are consistent with this objective. At the same time, if a culture of strong conformance to work procedures exists, then employees will want to operate with high patient safety while doing things the way they are designed to be done. When defects occur, it is clear that the concern for patient safety and the desire to conform to existing ways of operating will be in conflict. The defect vividly demonstrates that conformance is not good enough, and patient safety is compromised. Therefore, employees in this type of hospital cultural setting will be highly motivated to initiate root-cause repairs to processes, so that they can work in an environment where the processes are designed well for patient safety and they can therefore be followed as designed, without deviation.

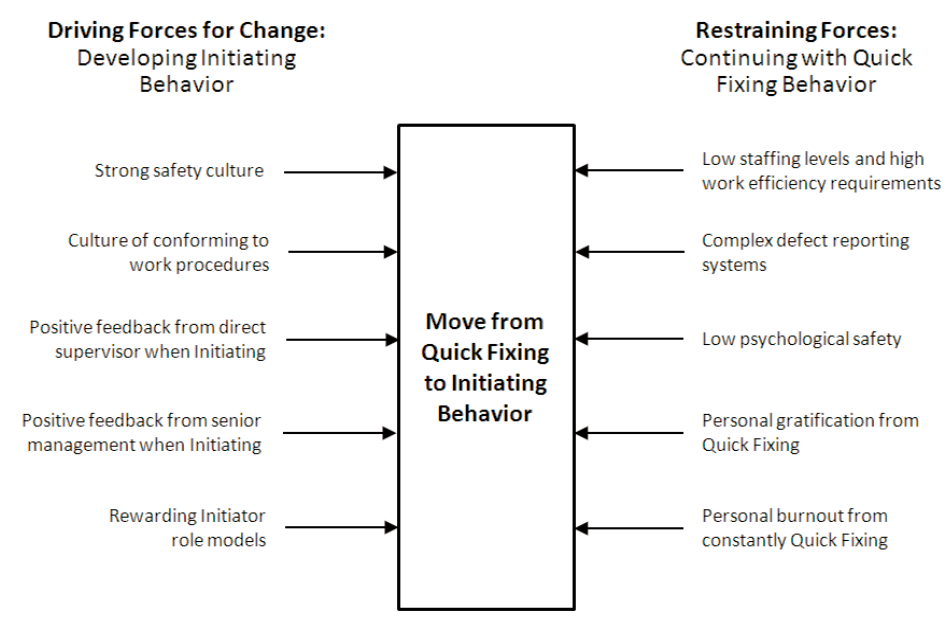

Figure 4. Making the transition from quick fixing to initiating behavior. 
The next two driving forces in Figure 4 are related to management feedback. Employees need to know their efforts to make improvements are valued, both by their direct supervisors and by more senior management in the hospital. If this feedback is clear and unambiguous, employees are more likely to invest in the effort of initiating and making improvements that are significant and long lasting, by attacking the root causes of the defects.

The fifth and last driving force has to do with incentives. Healthcare frontline professionals need role models: people at their level of the organization, who have become Initiators and are successful in their jobs and careers. Further, they need to see that Initiating behavior is rewarded. The exact nature of the reward, whether money, title, or other non-monetary recognition, is not as important as the fact that the reward was meaningful to the recipient and valued by the organization. This creates tangible examples for others to emulate.

While the driving forces for positive change were seen as significant by participants in this research, they also identified a number of forces that act as barriers to the transition to Initiating behavior. The top two forces on the right side of Figure 4 are organizational in nature, while the lower three are more personal in nature.

If employees are to become initiators of root-cause improvement when defects occur, they first must have the time in their work days to take the necessary actions for initiating real improvement. If staffing levels are low and the demands for efficiency and productivity are high, the perception is that there is not sufficient time to institute real improvements. In other words, through their staffing decisions, senior management has sent a clear signal that there is little time for reflection and consideration of ways to conceptualize and initiate long lasting improvements. Instead, making quick fixes and moving on is more likely to happen. Also, even if there is sufficient time in the employee's work schedule to push toward real improvement, if the defect reporting systems are too administratively burdensome, then employees will not be inclined to initiate real changes. Instead, they may be more likely to look for shortcuts and workarounds that solve the immediate problem but do not address the root causes of the problem.

Along with organizational restraining forces, there are three other forces in Figure 4 identified more closely with the employee's personal perceptions. Concerns related to psychological safety - an individual's ability to think and act freely without fear of negative consequences to self-image, status, or career - were repeatedly identified as a significant restraining force.

Prior research [49-50] has demonstrated that higher psychological safety increases the willingness of individuals to engage in error reporting and improvement behaviors, even though such efforts are inherently risky and can have negative personal and career consequences. Being associated with problems and change efforts can result in damage to one's reputation, particularly if the attempts to make positive changes are not successful. Therefore, workers will be more likely to engage in improvement efforts if they feel they have some protection from such backlash [50]. While the participants in this research used a range of terms and 
language to describe it, the fear was all too common that they will be professionally harmed or punished for making defects visible to the organization and escalating them for action. Hence, it was often seen as more expedient to keep defect resolution localized and low profile.

The last two restraining forces, gratification and burnout, come about because of the effects of taking Quick Fixing behavior. When an individual employs a quick fix approach to dealing with defects, he/she is naturally going to find some satisfaction in making the immediate instance of the problem go away. While the problem may come back again in the future, for now it is solved. This feeling of instant gratification can be a powerful motivator for continuing to use Quick Fixing to get rapid resolution of problems [49, 60]. It is tied to having a bias for action, which is often seen in organizations as a positive personal attribute. These feelings of personal gratification work against making the additional investments in time and energy required for root cause, permanent improvement.

While gratification comes immediately from undertaking quick fixes, a more insidious restraining force arises over time because of personal burnout [49, 60]. Depending on the employee's personal history of attempting to initiate real, long lasting improvements, he/she may have a history filled with frustration and failure. If the employee's past is negative in this regard, he/she may have already given up trying to initiate substantive improvements in operating conditions. Instead, the employee is resigned to putting patches on problems and dealing with defects on a one-off basis as they occur. Firefighting behavior becomes the norm, and the desire for true improvement is dampened. We have seen indications of improvement burnout in some of the most experienced employees, as they related their histories of trying and failing to make improvements.

\subsubsection{Challenge \#2: Developing Enhancing Behavior}

The second challenge of creating Enhancing behavior can be thought of as a continuous improvement challenge for the hospital and its leadership. To pose the challenge in the form of a question, it asks: When systems are working reasonably well and work processes are being followed, how can healthcare frontline professionals be motivated to take action to make further operating improvements?

In a sense, this continuous improvement challenge is more difficult than the defect challenge. Through our observations, discussions, and focus groups, we found that the challenge is actually twofold. First, how can employees become motivated to take any improvement action when the status quo is not overtly broken? In the absence of defects, how can a sense of improvement urgency be fostered and maintained? And second, given that employees are compelled to take improvement action, how can they be enticed and guided to take the right type of action?

Departing from Conforming behavior - As before, we used force field analyses to delineate between driving and restraining forces for this two-part challenge. Figure 5 presents the results of our data analysis for the first part, of transitioning employees from status quo, or Conforming, behavior to being motivated to take improvement action proactively in the absence of defects. 


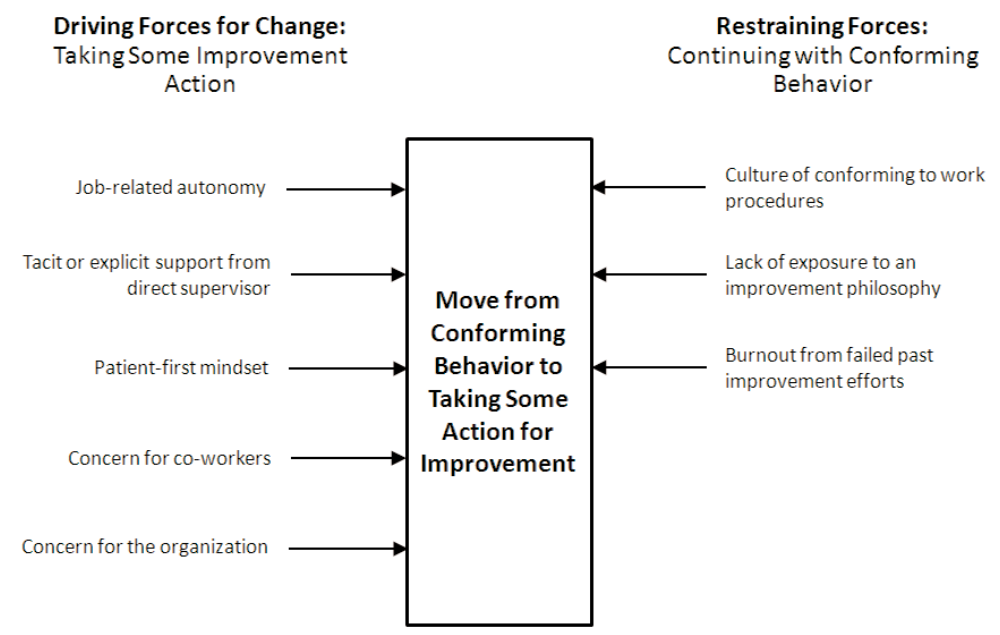

Figure 5. Departing from conforming behavior.

Figure 5 presents a set of driving forces for moving away from Conforming behavior. The top force, job-related autonomy, was found to be fundamental to making the transition to improvement action. Autonomy was cited [48] as a prerequisite for taking any out-of-the-norm action or deviating from standard operating procedures. Without a sense of autonomy, people will tend to follow the rules, especially if the rules are perceived to be good enough and not openly causing harm. Without autonomy, compliance is seen as a safe choice. On the other hand, higher levels of job autonomy can combine with another driving force - support from the employee's direct supervisor - to create an appealing work environment for continuous improvement. This support does not have to be mandated by the organization or even explicitly stated by the supervisor. We found that, as long as employees felt that supervisory support for improvement actions existed, they were empowered to take action.

Three other driving forces were found to be more personal in nature. The lower three driving forces in Figure 5 all point to individualized attributes of the healthcare employee. We found that those individuals with a strong sense of care and concern for patients, co-workers, and the organization were more likely to break free from the status quo to take improvement actions. They want to work in an environment of excellence, which compels them to make improvements.

Playing against these driving forces are three restraining forces on the right side of the figure. The first force is a culture of conforming to work procedures. Note that this same force was seen by our research participants as a driving force in Figure 4, when defects occur. This highlights the difference in desired behavior in the presence of defects versus the absence of defects. Referring back to Figure 4, defects are tangible evidence that processes are broken. Because of the desire to conform to work procedures, employees will be motivated to make substantive process improvement so that, after the improvement, they can once again feel comfortable complying with the 
updated work procedures. Conversely, in Figure 5's defect-free environment, a desire to comply with work procedures will act to motivate healthcare professionals to keep doing their jobs the same way as they have always done them.

The other two restraining forces listed in Figure 5 are related to knowledge to improve and desire to improve, respectively. Concerning the knowledge to improve, if healthcare professionals have not been exposed to an improvement philosophy at work, they are less likely to know the mechanics of how to continuously improve. While they are skilled and experienced in their medical and clinical specialties, this does not necessarily mean they are proficient at process and systems improvement. The specialized methods, tools, and mindset required to succeed at continuous improvement must be disseminated to frontline workers, which requires a dedicated effort and sustained commitment by senior management.

For desire to improve, we found that worker burnout once again comes into play. Previous efforts to improve that have failed are a strong de-motivator to individuals [49, $60,61]$. This is especially true in the absence of defects, when current conditions do not require improvement intervention. To create a culture of continuous improvement, hospital leadership must address the burnout issue and find ways to reenergize those employees who have tried and failed in the past.

Transitioning to Enhancing behavior - As discussed above, there are a number of forces that either promote or inhibit the change from Conforming behavior to taking improvement action. In addition to these forces, our research uncovered another set of forces that affect the willingness of healthcare professionals to take the right type of improvement action by exhibiting Enhancing behavior. Figure 6 presents this expanded set of relevant forces.

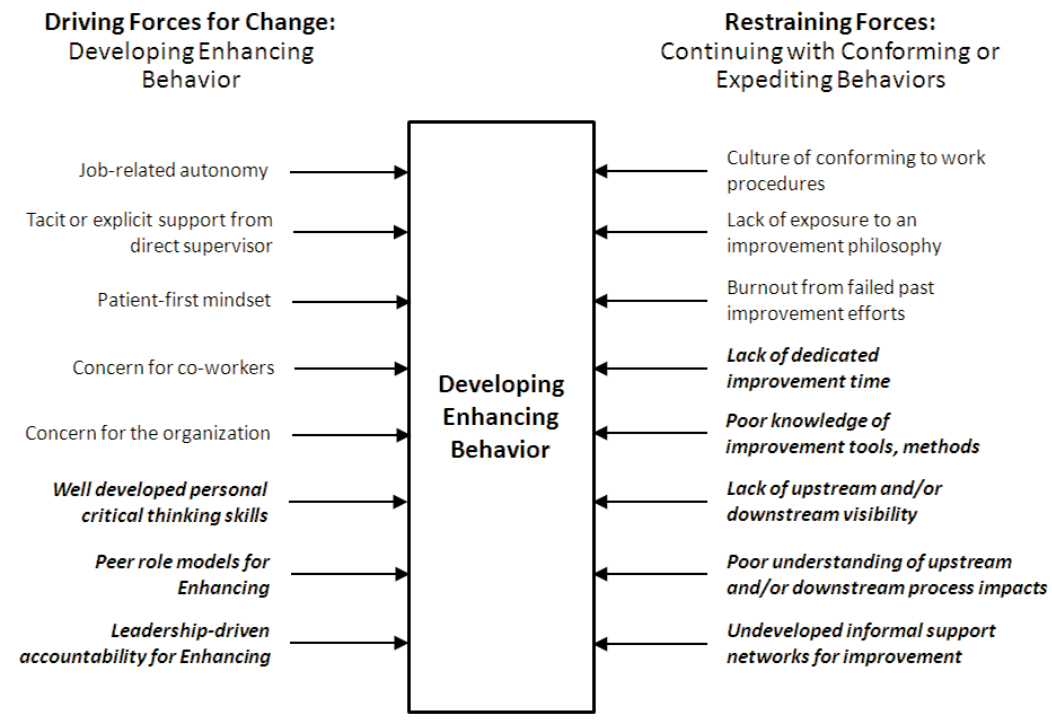

Figure 6. Transitioning to enhancing behavior. 
Figure 6 builds upon Figure 5 by identifying additional forces conducive to the desired state of Enhancing behavior. As noted earlier, developing Enhancing behavior is a two-part challenge. Improvement action alone is not enough; the right type of improvement action is also necessary. Compared to Figure 5, the additional forces in Figure 6 are displayed in bold italics and are discussed below.

Three driving forces were found to be important in making the transition to Enhancing behavior, and are displayed on the lower left side of Figure 6. The first of these is critical thinking skills. Critical thinking is higher order thinking whereby an individual skillfully conceptualizes and assesses situations, questions assumptions, determines options for responding to the situation, and makes intelligent decisions. In the context of process improvement, critical thinking includes the ability to analyze operating conditions to understand the opportunities for real improvement, to reflect on the actions required to accomplish this improvement, to understand the associated organizational and personal expectations, and to make an internal commitment to making the improvement happen. In our research, we have found that Enhancing behavior is associated with high critical thinking skills. An enhancer knows what it takes to drive continuous improvement and is committed to this course of action.

The second driving force for developing Enhancing behavior is that of having peer role models. Role models can provide guidance by example, offer advice and feedback, and serve as visible examples of the type of organizational behavior that is valued. The final driving force of Figure 6 is having a hospital leadership team that holds the organization and its employees accountable for becoming a continuous improvement organization. Most of the time, committed professionals will rise to meet the level of expectations set for them. It is leadership's role to set improvement expectations at a high but attainable level. In the absence of defects, leadership must develop this sense of urgency to improve and communicate its importance. A comment from an executive leader in one of the rural hospitals captures this sentiment:

"My role is to maintain the rigor on sustaining improvement results. I devote extra time and attention to work with employees who engage in improvement."

Moving to the right side of Figure 6, there are five additional restraining forces that emerged during our data collection efforts. The first is lack of dedicated improvement time. Without the time to invest in Enhancing behavior, employees will find it difficult to sustain this type of behavior over the long run. In our research, we observed two different approaches to allocating improvement time. One way, seen in the hospital where we observed medication delivery processes, is to reduce individual workloads on more of a piecemeal basis. In the absence of a formal continuous improvement program, employees throughout the hospital allocated time for improvement that was subject to the constantly varying workload conditions in their units and departments. Alternately, the four rural hospitals in our study all had formal continuous improvement programs in place. This allowed employees to allocate consistent amounts of time to improvement, as the programs had their own budgets and objectives that needed to be met. While the formal program approach has obvious advantages, we have observed that either way can be effective if sufficient time is made available. 
Similar to the lack of time, another restraining force is the lack of knowledge of improvement tools and methods. There is a body of knowledge on improvement that incorporates insights and techniques from industrial engineering, business management, psychology, and leadership. Those who are effective at enhancing need to be trained in these areas and build experience over time in applying this knowledge. The need for formal training programs and learning by doing activities is well accepted in many industries, and is increasingly becoming the norm in healthcare delivery.

The next two restraining forces are related to a bigger picture view of how improvement actions can touch all parts of the hospital. Hospitals are tightly coupled operating organizations, where the actions of one unit, department, or area tend to affect or be affected by actions in other units, departments, and areas. For employees to exhibit Enhancing behavior, they need visibility of how their local work areas are connected to the larger operating system. According to one leader:

"I expect myself and others to use A3 thinking [A3 thinking is a standard Lean approach and tool used for defining opportunities or problems and then taking action]. It allows us to have a common systems view. It makes it easy for employees to engage in operating discussions, and they can easily understand what other areas of the hospital are doing."

Along with this visibility, they need an understanding of how the impacts of improvement actions can cross organizational boundaries and have either positive or negative effects.

"Our methods and mechanisms for executing improvement ideas give our employees a way to collaborate across the organization and get things done."

Without sufficient visibility and understanding, it is difficult for employees to be the source of root-cause, system-wide improvements.

The fifth and last restraining force focuses on the employee's social network within the hospital. Outside of senior leadership, direct supervision, and explicit continuous improvement role models, a potential enhancer needs a support system of colleagues. Employees who strive to enhance will have inevitable setbacks and challenges along the way. The support and encouragement of colleagues can prove to be invaluable to staying the course. A leader's comment demonstrates recognition of this fact:

"I honestly cannot imagine what we would be doing without our performance improvement engine [referring to Lean]. It gives us and our people a way to go from point A to point B. They collaborate without our permission and support each other when times get tough."

\section{DISCUSSION}

In summary, our observations and findings suggest that individuals exhibiting Quick Fixing, Conforming, and Expediting behaviors are affected by factors that are tied to single-loop learning. They behave in a manner that acts against the organization's long- 
term interests and may even cause healthcare delivery systems to produce more defects over time. A summary of the actions that organizations can take to address such managerial challenges are described below.

Those with formal power in the organization can take positive actions in many of the areas described above to increase Initiating and Enhancing behaviors and reduce Quick Fixing, Conforming, and Expediting behaviors. Our observations indicate that healthcare professionals closely watch and examine behaviors and feedback from their leaders and immediate managers. We believe that leadership teams willing to create organizations that operate with a double-loop learning mindset must wield social influence and become referents for the employees regarding quality improvement efforts. Research has shown that a psychologically safe climate must be cultivated by leaders and managers if frontline employees are to begin to report defects and initiate improvements [62-70]. Accordingly, we believe that management involvement, style, and explicit behavior play important roles in organizational change. Perhaps here lies a critical step in the journey of developing learning organizations: the need for leaders and managers to "look in the mirror" and admit that that they are responsible for developing improvement behaviors, and therefore they must build organizations with values and a purpose beyond Quick Fixing, Conforming, and Expediting.

In addition, we want to stress the importance of leadership's willingness to hold the organization accountable for improvement or lack thereof. The leadership team needs to support the agreed upon solutions and new ways of operating that result from Initiating and Enhancing behaviors. At the same time, when there are non-compliant behaviors that are problematic, leadership must hold those persons accountable and enforce discipline when necessary. For example, Furman and Caplan [71] provided a description of the Virginia Mason Medical Center patient safety alert system that, in five years, led to intensive safety counseling interventions and, eventually, suspension of over 50 staff and physicians for unsafe behavior. Accountability cuts both ways, and a strong culture of accountability sends a clear signal to all employees that process and systems improvement is required and expected.

Even though enlightened leadership is a critical component in nurturing an improvement culture, the transition from single-loop to double-loop learning is not something that can be mandated from the top of the organization. Leadership teams can only do so much; it is also up to each individual to make the internal commitment to become a valuable contributor in the face of defects and deep seated problems with processes and ways of performing work. Such work occurs over a prolonged period of time, and depends upon each employee's understanding, motivation, abilities, and desire to make constructive changes in his/her work environment [72-73]. Because Initiating and Enhancing behaviors are usually difficult if attempted by one isolated employee, a good example of a coordinated, collaborative activity that helps employees accomplish such behaviors is the multiday, multi-disciplinary rapid improvement event. Participation in these events and other improvement activities will build a sense of confidence in employees that they can make a difference. 
From the results of this research, we are inclined to believe that the motivational gap between single-loop and double-loop learning behavior for many employees is relatively small. Our observations strongly support the position that, by and large, healthcare professionals take pride in their jobs and care deeply about patient safety and positive patient outcomes. The larger gap exists in developing systems, mechanisms, and policies that support employees in the double-loop learning journey. For instance, this includes defect reporting and improvement tracking systems that are efficient and do not waste valuable employees' time that could be used to provide patient care. It also includes rapid, well thought out feedback about the results and impacts of employee improvement suggestions. Therefore, quality improvement behaviors, systems, and mechanisms all have to be aligned. Some hospitals have already implemented such systems and mechanisms with success [74], but there is a long way to go. Nonetheless, it is encouraging that the identified factors and managerial challenges in this research are relatively similar to those found in studies that used theories of quality improvement implementation and organizational change [31-37].

There are several limitations to this study, and thus caution should be exercised in generalizing our findings. First, the results are based on a qualitative study of a limited number of observations and participants. In phase 1, we studied instances of behaviors collected by one researcher, and did not track behaviors by individuals over time. New samples with different characteristics, such as different patient and employee demographics, different types of hospital services provided, and especially the tracking of specific individuals' behaviors, may yield new insights. Second, hospital employees may behave differently because they are subjected to observation [75-76]. Third, observers were not blinded and thus an unintentional bias is possible. Finally, in studies such as the current one that involves understanding the meaning of a broad range of factors related to quality improvement, designing study protocols and translating observations into data are not easy [77]. We were required to make judgments when categorizing factors, as the participants' language was sometimes unclear and not supported by definitions explicitly stated in the literature. Therefore, we tried to provide explanations for the improvement behaviors and drivers to allow readers to consider these issues in context. We believe that future research would benefit from clear definitions of factors and continued study of these behaviors and drivers.

\section{CONCLUSION}

A key contribution of this research is the identification of five types of improvement behaviors. The current study also presents a unique set of driving and restraining forces that may affect employees' improvement behaviors, which in turn may have significant implications for hospital improvement programs and for healthcare engineering practitioners and researchers. This begins to fill the gap in knowledge about what factors drive effective improvement efforts in hospital settings.

Finally, we believe that as improvement practitioners focus on helping healthcare employees make the transition to double-loop learners and root-cause problem solvers, 
the rates of effective improvement efforts will be greatly increased and patient safety and system efficiencies will be much improved. If successfully applied, our findings may help counterbalance the negative effects of employee frustrations and burnout regarding improvement efforts, possibly resulting in higher retention rates of healthcare professionals. These efforts will help hospitals achieve breakthrough results in quality, safety, cost reduction, and patient satisfaction as envisioned by the Institute of Medicine [4].

\section{ACKNOWLEDGMENTS}

The authors are grateful to all nurses, technicians, managers and leaders who participated in this research. We would also like to acknowledge Dr. Marianne Jackson for her valuable insights, and Akshay Venkitasubramanian for his help with literature review.

\section{CONFLICT OF INTEREST}

The authors indicated no potential conflict of interest.

\section{REFERENCES}

[1] Institute of Medicine. To Err is Human: Building a Safer Health System, A Report of the Institute of Medicine. Washington, DC, 1999.

[2] Aiken LH, Clarke SP, Sloane DM. An international perspective on hospital nurses' work environments: The case for reform. Policy, Politics, and Nursing Practice. 2001, 2(4):255-63.

[3] Aiken LH, Clarke SP, Sloane DM, Sochalski J, Silber JH. Hospital nurse staffing and patient mortality, nurse burnout, and job dissatisfaction. Journal of American Medical Association. 2002, 288(16):1987-1993.

[4] Institute of Medicine. Crossing the Quality Chasm: A New Heath Care System for the $21^{\text {st }}$ Century, A Report of the Institute of Medicine, Washington, DC, 2001.

[5] Institute of Medicine. Building a Better Delivery System: A New Engineering / Health Care Partnership, A Report of the Institute of Medicine, Washington, DC, 2005.

[6] Institute of Medicine. Preventing Medication Errors: Quality Chasm Series, A Report of the Institute of Medicine, Washington, DC, 2006.

[7] Blumenthal D, Kilo CM. A report card on continuous quality improvement. The Milbank Quarterly. 1998, 76(4):625-648.

[8] Batalden PB, Davidoff F. What is 'quality improvement' and how can it transform healthcare. Quality and Safety in Health Care. 2007, 16(1):2-3.

[9] Leape L, Berwick D, Clancy C, Conway J, Gluck P, Guest J, Lawrence D, Morath J, O'Leary D, O’Neill P, Pinakiewicz D, Isaac T. Transforming health care: A safety imperative. BMJ Quality and Safety. 2009, 18(6):424-428.

[10] Chassin MR, Becher EC. The wrong patient. Annals Internal Medicine, 2002, 136(11):826-833.

[11] Berwick D. A user's manual for the IOM's "Quality Chasm” report. Health Affairs, 2003, 21(3):80-90.

[12] Sexton JB, Thomas EJ, Helmreich RL. Error, stress, and teamwork in medicine and aviation: Cross sectional surveys. British Medical Journal. 2000, 320:745-749.

[13] Thomas EJ, Sexton JB, Neilands TB, Frankel A, Helmreich RL. The effect of executive walk rounds on nurse safety climate attitudes: A randomized trial of clinical units. BMC Health Services Research. 2005, 5(28):1-9.

[14] Anderson JG, Ramanujam R, Hensel D, Anderson MM, Sirio CA. The need for organizational change in patient safety. International Journal of Medical Informatics. 2006, 75(12):809-817. 
[15] Ramanujam R, Rousseau DM. The challenges are organizational, not just clinical. Journal of Organizational Behavior. 2006, 27(7):811-827.

[16] McFadden KL, Stock GN, Gowen CR. Exploring strategies for reducing hospital errors. Journal of Healthcare Management. 2006, 51(2):123-136.

[17] McFadden KL, Towell ER, Stock GN. Implementation of patient safety initiatives in US Hospitals. International Journal of Operations and Production Management. 2006, 26(3):326-347.

[18] Wauben LSGL, Lange JF, Goossens RHM. Learning from aviation to improve safety in the operating room - a systematic literature review. Journal of Healthcare Engineering. 2012, 3(3):373-390.

[19] Leveson N, Couturier M, Thomas J, Dierks M, Wierz D, Psaty BM, Finkelstein S. Applying system engineering to pharmaceutical safety. Journal of Healthcare Engineering. 2012, 3(3):391-414.

[20] Reason J. Human Error, Cambridge University Press, Cambridge, NY, 1990.

[21] Reason J. Beyond the organizational accident: The need for 'error wisdom' on the frontline. Quality and Safety in Health Care. 2004, 13(1):28-33.

[22] Roberts KH. Some characteristics of high reliability organizations. Organization Science. 1990, 1(2):160-177.

[23] Roberts KH, Bea R. Must accidents happen? Lessons from high reliability organizations. Academy of Management Executive. 2001, 15(3):70-79.

[24] Chassin MR, Loeb MJ. The ongoing quality improvement journey: Next stop, high reliability. Health Affairs. 2011, 30(4):559-568.

[25] Spear SJ, Bowen HK. Decoding the DNA of the Toyota Production System. Harvard Business Review. 1999, 77(5):97-106.

[26] Thompson DN, Wolf GA, Spear SJ. Driving improvement in patient care. Journal of Nursing Administration. 2003, 33(11):585-595.

[27] Shortell S. Increasing value: A research agenda for addressing the managerial and organizational challenges facing health care delivery in the United States. Medical Care Research and Review. 2004, 61(3):12-30.

[28] Weingart SN, Page D. Implications for practice: Challenges for healthcare leaders in fostering patient safety. Quality and Safety in Health Care. 2004, 13(6):52-56.

[29] Grol R, Bosch M, Hulscher M, Eccles M, Wensing M. Planning and studying improvement in patient care: The use of theoretical perspectives. Milbank Quarterly. 2007, 85(1):93-138.

[30] Classen DC, Resar R, Griffin F, Federico F, Frankel T, Kimmel N, Whittington JC, Frankel A, Seger A, James BC. Global trigger tool shows that adverse events in hospitals may be ten times greater than previously measured. Health Affairs. 2011, 30(4):581-589.

[31] Dixon-Woods M, Bos CL, Aveling EL, Goeschel CA, Pronovost P. Explaining Michigan: Developing an ex-post theory of a quality improvement program. Milbank Quarterly. 2011, 89(2):167-205.

[32] Weiner BJ, Alexander JA, Baker LC, Shortell SM, Becker M. Quality improvement implementation and hospital performance on patient safety indicators. Medical Care Research and Review. 2006a, 63(1):29-57.

[33] Weiner BJ, Alexander JA, Shortell SM, Baker LC, Becker M, Geppert JJ. Quality improvement implementation and hospital performance on quality indicators. Health Services Research. 2006b, 41(2):307-34.

[34] Alexander JA, Weiner BJ, Shortell SM, Baker LC, Becker MP. The role of organizational infrastructure in implementation of hospitals' quality improvement. Hospital Topics. 2006, 84(1):11-20.

[35] Rachel M, Werner RM, Kolstad JT, Stuart EA, Polsky D. The effect of pay-for-performance in hospitals: Lessons for quality improvement. Health Affairs. 2011, 30(4):690-698.

[36] Solomons NM, Spross JA. Evidence-based practice barriers and facilitators from a continuous quality improvement perspective: An integrative review. Journal of Nursing Management. 2011, 19(1):109-120. 
[37] Shortell SM, Bennett CL, Byck GR. Assessing the impact of continuous quality improvement on clinical practice: What it will take, Milbank Quarterly. 1998, 76(4):593-624.

[38] Kaplan HC, Brady PW, Dritz MC, Hooper DK, Linam WM, Froehle CM, Margolis P. The influence of context on quality improvement success in health care: A systematic review of the literature. Milbank Quarterly. 2010, 88(4):500-559.

[39] Kaplan HC, Pronovost LP, Froehle CM, Margolis P. The model for understanding success in quality (MUSIQ): Building a theory of context in healthcare quality improvement. BMJ Quality and Safety. 2011, 21(1):13-20.

[40] Reid J, Catchpole K. Patient safety: A core value of nursing - so why is achieving it so difficult? Journal of Research in Nursing. 2011, 16(5):209-223.

[41] Perkins MB, Jensen PS, Jaccard J, Gollwitzer P, Oettingen G, Pappadopulos E, Hoagwood KE. Applying theory-driven approaches to understanding and modifying clinicians' behavior: What do we know? Psychiatric Services. 2007, 58(3):342-348.

[42] Davidoff F. Heterogeneity is not always noise: Lessons from improvement. Journal of American Medical Association. 2009, 302(23):2580-2586.

[43] Bandura A. Social cognitive theory of self-regulation. Organizational Behavior and Human Decision Processes. 1991, 50(2):248-287.

[44] Argyris C, Schon D. Organizational Learning: A Theory of Action Perspective, Addison-Wesley, Reading, MA, 1978.

[45] Davies HT, Nutley SM. Developing learning organizations in the new NHS. British Medical Journal. 2000, 320(7740):998-1001.

[46] Ferlie EB, Shortell SM. Improving the quality of health care in the United Kingdom and the United States: A framework for change, Milbank Quarterly. 2001, 79(2):281-315.

[47] Huq Z, Martin T N. Workforce cultural factors in TQM/CQI implementation in hospitals. Health Care Management Review. 2000, 25(3):80-93.

[48] Edmondson AC. Learning from mistakes is easier said than done: Group and organizational influences on the detection and correction of human error. Applied Behavioral Science. 1996, 32(1):5-28.

[49] Tucker AL, Edmondson AC. Why hospitals don't learn from failures: Organizational and psychological dynamics that inhibit system change. California Management Review. 2003, 45(2):55-72.

[50] Edmondson AC. Psychological safety and learning behavior in work teams. Administrative Science Quarterly. 1999, 44(2):350-383.

[51] Laschinger HKS, Shamian J, Thomson D. Impact of magnet hospital characteristics on nurses' perceptions of trust, burnout, quality of care, and work satisfaction. Nursing Economics. 2001, 19(5):209-219.

[52] Lee H, Song R, Cho YS, Lee GZ, Daly B. A comprehensive model for predicting burnout in Korean nurses. Journal of Advanced Nursing. 2003, 44(5):534-545.

[53] Janz BD, Colquitt JA, Noe R. Knowledge worker team effectiveness: The role of autonomy, interdependence, team development, and contextual support variables. Personnel Psychology. 1997, 50(4):877-904.

[54] Whitley MP, Putzier DJ. Measuring nurses' satisfaction with the quality of their work and work environment. Journal of Nursing Care Quality. 1994, 8(3):43-51.2-3.

[55] Mazur LM, McCreery JK, Rothenberg L. Facilitating lean learning and behaviors in hospitals during the early stages of lean implementation. Engineering Management Journal. 2012, 24(1):36-47.

[56] Mazur LM. The study of errors, expectations, and skills for medication delivery improvement. $\mathrm{PhD}$ dissertation, Montana State University, 2008.

[57] Strauss A, Corbin J. Basics of Qualitative Research: Grounded Theory Procedures and Techniques, Sage Publications, Newbury Park, CA, 1998.

[58] Wagner HT, Morton SC, Dainty ARJ, Burns ND. Path dependent constraints on innovation programmes in production and operations management. International Journal of Production Research. 2011, 49(11):3069-3085. 
[59] Hayden Jr WM. Human systems engineering, A trilogy, Part II: May the force be with you: Anatomy of project failures. Leadership \& Management in Engineering. 2006, 6(1):1-12.

[60] Dutton JE. The making of organizational opportunities: An interpretive pathway to organizational change, In L. L. Cummings, \& B. M. Staw (Eds.), Research in organizational behavior (pp. 195-226). Greenwich, CT: JAI Press, 1993.

[61] Repenning NP, Sterman JD. Capability traps and self-confirming attribution errors in the dynamics of process improvement. Administrative Science Quarterly. 2002, 47(2):265-295.

[62] Kahn WA. Psychological conditions of personal engagement and disengagement at work. Academy of Management Journal. 1990, 33(4):692-724.

[63] Senge PM. The Fifth Discipline: The Art \& Practice of The Learning Organization, New York: Doubleday Currency, 1990.

[64] Kerfoot K. Staff engagement: It starts with the leader. Medsurg Nursing: Official Journal of the Academy of Medical-Surgical Nurses. 2008, 17(1):64-65.

[65] McAlearney AS. Leadership development in healthcare: A qualitative study. Journal of Organizational Behavior. 2006, 27(7):967-982.

[66] Kotter JP. Leading Change, Harvard Business School Press, 1996.

[67] Nembhard IM, Edmondson AM. Making it safe: The effects of leader inclusiveness and professional status on psychological safety and improvement efforts in health care teams. Journal of Organizational Behavior. 2006, 27(7):941-966.

[68] Tucker AL. An empirical study of system improvement by frontline employees in hospital units. Manufacturing and Service Operations Management. 2007, 9(4):492-505.

[69] Ghoshal S, Bartlett GA. Linking organizational context and managerial action: The dimensions of quality of management. Strategic Management Journal. 1994, 15(S2):91-112.

[70] Koys D. The effects of employee satisfaction, organizational citizenship behavior, and turnover, on organizational effectiveness: A unit-level, longitudinal study. Personnel Psychology. 2001, 54(1):101-114.

[71] Furman C, Caplan R. Applying the Toyota Production System: Using a patient safety alert system to reduce error. The Joint Commission Journal on Quality and Patient Safety. 2007, 33(7):376-386.

[72] Lapre MA, Mukherjee AS, Wassenhove LNV. Behind the learning curve: Linking learning activities to waste reduction. Management Science. 2000, 46(5):597-611.

[73] Lapre MA, Wassenhove LNV. Creating and transferring knowledge for productivity improvement in factories. Management Science. 2001, 47(10):1311-1325.

[74] Institute of Healthcare Improvement. Going Lean in Health Care, 2005 (available at http://www.ihi.org/knowledge/Pages/IHIWhitePapers/GoingLeaninHealthCare.aspx; last accessed on August 2012).

[75] Adair G. The Hawthorne effect: A reconsideration of the methodological artifact. Journal of Applied Psychology. 1984, 69(2):334-345.

[76] Gilovich T, Griffin D, Kahneman D. Heuristics and Biases: The Psychology of Intuitive Judgment, Cambridge University Press, Cambridge, UK, 2002.

[77] Sharita J, McCane L, Schulman CI, Graygo JM, Augenstein JS. The use of observation and interview methods for assessing issues in patient care in the resuscitation unit of a level-1 trauma center. Journal of Healthcare Engineering. 2010, 1(1):83-100.

\section{APPENDIX A - INTERVIEW QUESTIONS IN PHASE 2}

\section{Questions for Leaders}

1. How has [hospital name]'s sense of urgency for developing desired improvement behaviors changed over the years? What do you anticipate happening with this sense of urgency going forward? Why? 
2. Do managers, physicians, nurses and other stakeholder groups feel psychologically safe and motivated to support your improvement efforts? If some are not, why is this the case? How are you dealing with these groups?

3. Over the past year or so, have you increased or decreased the pace of improvement in your organization? What tangible benefits and risks relate to this increase or decrease in speed?

4. Have you seen signs of improvement burnout in your hospital? Why?

5. What does a culture of continuous quality improvement mean to you, and when do you know you have created it at [hospital name]? Does it exist now? How do you know this? What could cause it to fail?

6. What have you been doing, and plan to do in the future, to ensure that people/groups understand "What's In It For Me?" concerning your improvement efforts?

7. How do you get people throughout the organization to actually engage in desired improvement behaviors and improve their operations?

8. Do you reward or penalize people/groups for good or poor improvement behaviors? How do you make this visible to employees throughout the hospital?

9. How do you deal with people/groups who are not willing to buy into an environment of improvement?

10. Can you provide an example or two on how frontline professionals have taken personal initiative and brought forward new improvement ideas?

11. What are you doing to develop a "human capital" network for improvement that reaches across the entire hospital?

12. What does real commitment to improvement mean to you? Has real commitment been established at [hospital name] in frontline professionals, middle management, and physicians? How do you know this?

13. What steps were taken to inform and educate hospital employees on improvement methods and desired improvement behaviors? Were these steps adequate? Were they effective?

14. Do employees understand how their improvement actions affect other parts of the hospital? How are you developing this capability in your employees?

15. Are there still key individuals in your hospital who do not support your human development efforts? If so, how do you plan to deal with them?

16. How will you ensure that the desired improvement behaviors continue to be used over time?

17. What mechanisms did you or will you put in place to make sure that your hospital doesn't fall back into the old ways of operating?

\section{Questions for Focus Groups}

1. How has leadership supported you during the transition to continuous quality improvement?

2. How were you trained on continuous quality improvement? Was this training adequate? Was it effective?

3. Do you feel psychologically safe and motivated to take actions for improvement? Why?

4. Have you been rewarded or penalized for taking improvement action?

5. What does a culture of continuous quality improvement mean to you? Does it exist now at [hospital name; your department]? How do you know this? What could cause it to fail? 
6. What's in it for you? Why should you get involved with improvement efforts?

7. Do you know people/groups who are not willing to buy into a culture of improvement? Why is this the case?

8. Are you burned out from trying to make improvements? Why?

9. Are others around you burned out from trying to make improvements? Why?

10. Can you provide an example or two on how you took the initiative to make an improvement? What was most challenging about this? What was most rewarding about this?

11. What will you do to not fall back into the old ways of operating? 



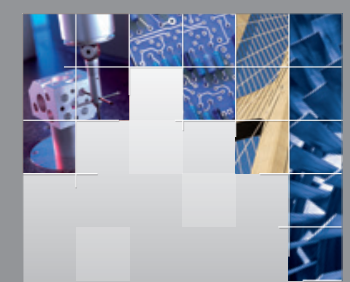

\section{Enfincering}
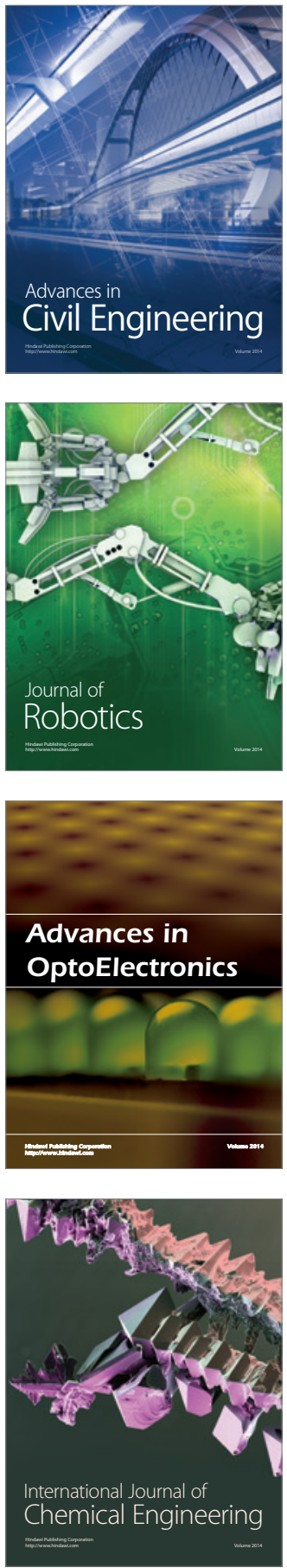

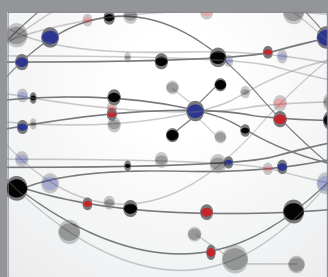

The Scientific World Journal

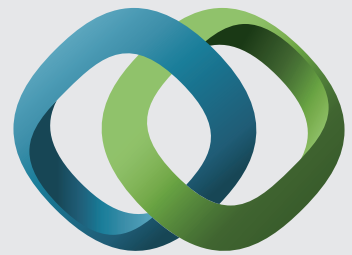

\section{Hindawi}

Submit your manuscripts at

http://www.hindawi.com
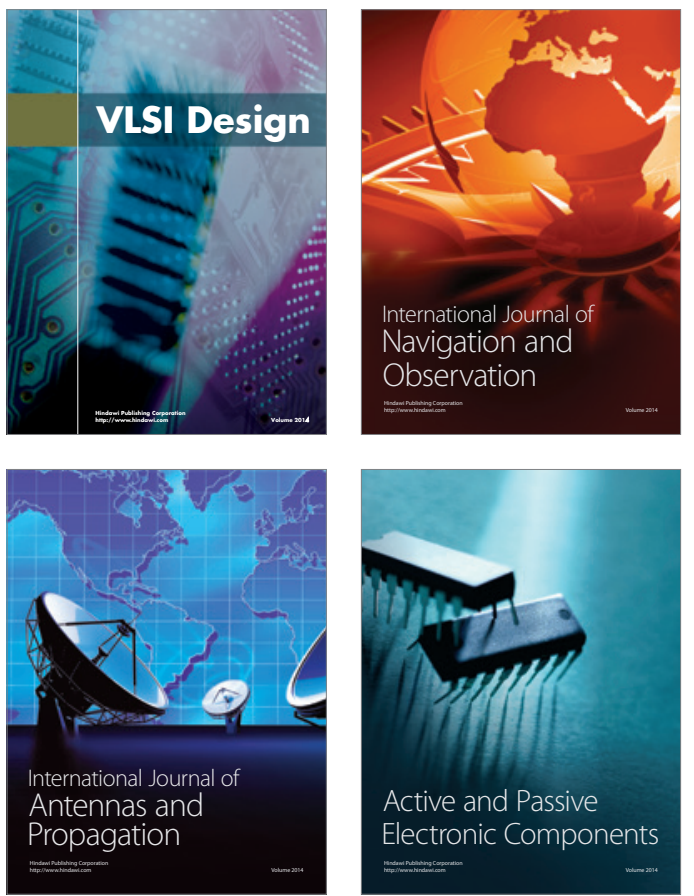
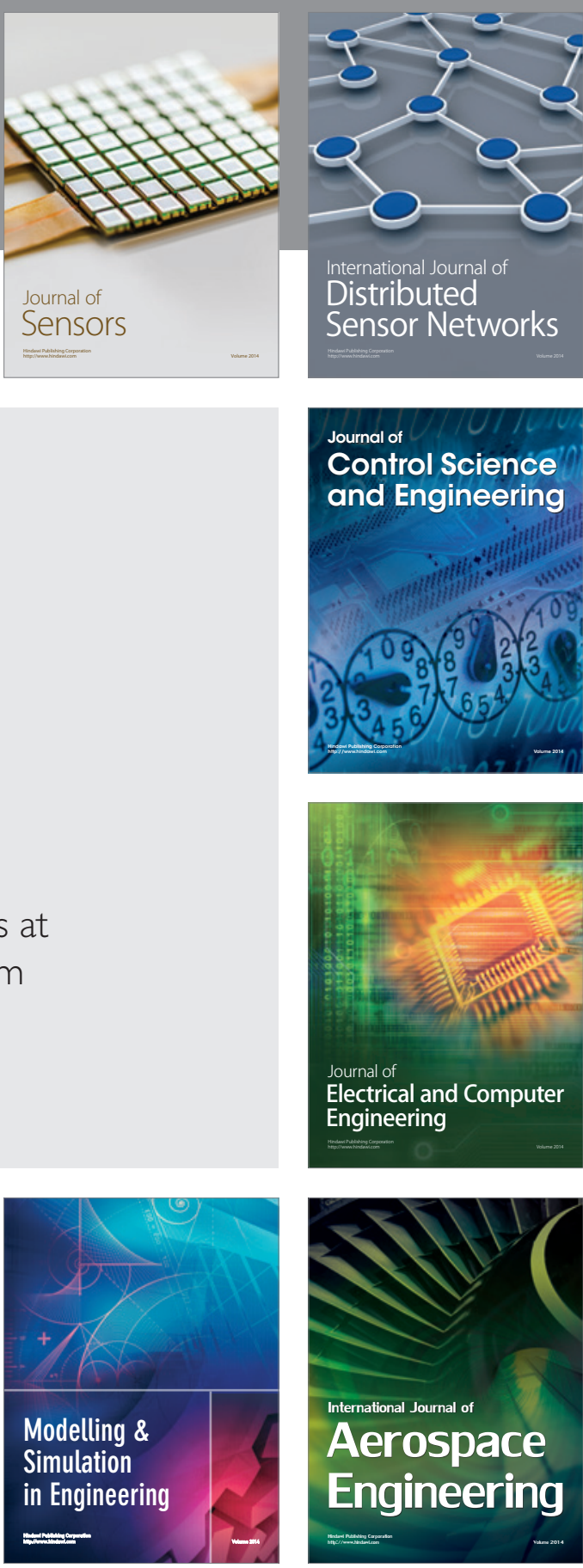

International Journal of

Distributed

Sensor Networks

Journal of

Control Science

and Engineering
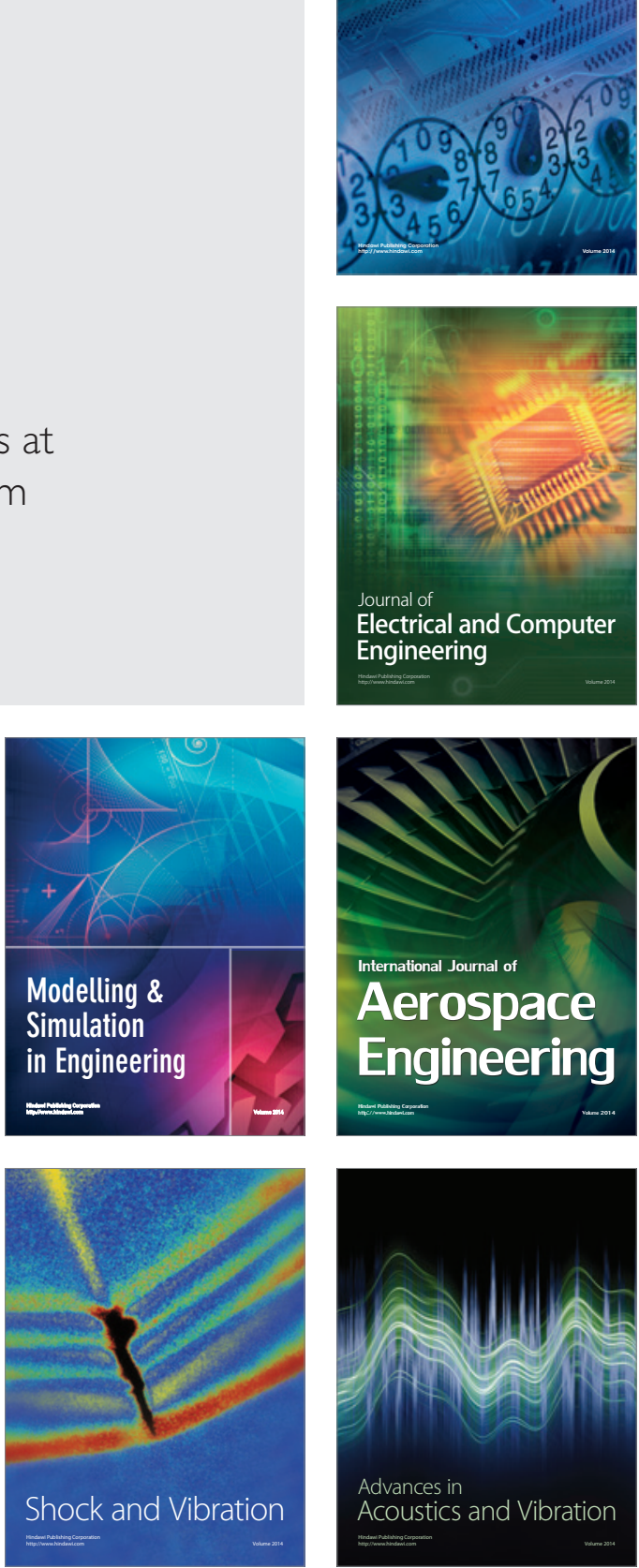